\title{
Data Fitting-based SNR estimation algorithm for the Adaptive Transmission
}

\author{
Sun Zezhou, Wang Chenghua, Zhang Xiaofei \\ College of Electronic and Information Engineering, Nanjing University of Aeronautics and
}

Astronautics, Nanjing, China, 210016

Email: zhangxiaofei@nuaa.edu.cn

Keywords: SNR estimation, adaptive transmission, parameter estimation, data fitting.

\begin{abstract}
Adaptive transmission system selects the suitable transmission rate on the basis of the current channel state. In this paper, we exploit the signal to noise ration (SNR) as a benchmark of standard to measure the channel state. We employ data fitting (DF) to estimate SNR, which is a key technology of the adaptive transmission, and we change the transmission rate according to the estimation of SNR. The proposed algorithm can obtain a low computational complexity and can have performance improvement of SNR estimation. The superiority of the proposed algorithm is revealed by simulations..
\end{abstract}

\section{Introduction}

Adaptive transmission technique is a significant method of judging the channel condition and adjusting the transmission rate of the system according to the stand or fall of the channel state [1-2]. Higher transmission rate is chosen with a good channel state while the data throughput will be degrading with the channel quality getting poor. In this paper, an adaptive transmission scheme is studied, which applies adaptive variable-rate technique to change the data rate of the communication system. In this paper, the signal to noise ration (SNR) is taken as the benchmark of channel condition. The receiver first estimates the SNR of the receiving signal in real time. According to the result of comparison between the SNR estimation and the give threshold, the receiver chooses a suitable transmission rate and gives a feedback to transmitter in the form of control information. Finally the transmitter gives a command to switch the rate while detecting the control information.

SNR estimation method consists of time domain method and frequency domain method. For the time domain method, it can be divided into data aided (DA) method and non-data aided (NDA) method [3]. DA method has a higher estimation accuracy than NDA method, but involving the insertion of periodic pilot sequence, which is inefficient. In time domain method, the SNR estimation algorithms based on DA include minimum mean square error (MMSE), maximum likelihood (ML), separating character matrix estimation (SSME) and high-order-cumulants signal-noise separation method, while the SNR estimation algorithms based on NDA contain M2M4, Signal-to-Variation Ratio (SVR) and squared Signal-to-Noise Variance (SNV) [5-12]. In this paper, we use Data Fitting (DF) method for SNR estimation, which is a key technology in field of adaptive transmission. The proposed algorithm can obtain a low computational complexity and can have performance improvement of SNR estimation. The superiority of the proposed algorithm is revealed by simulations. The reminder of this paper is structured as follows.

\section{The adaptive transmission scheme}

Adjusting the transmission rate based on SNR is a process that first detecting the present SNR value in real-time through the receiving signal, and then deciding the transmission rate according to the SNR value. Adaptive rate transmission based on SNR reflects the present channel state. In this 
paper, the adaptive rate transmission mechanism based on SNR is applied, and the schematic block diagram is shown in Fig. 1.

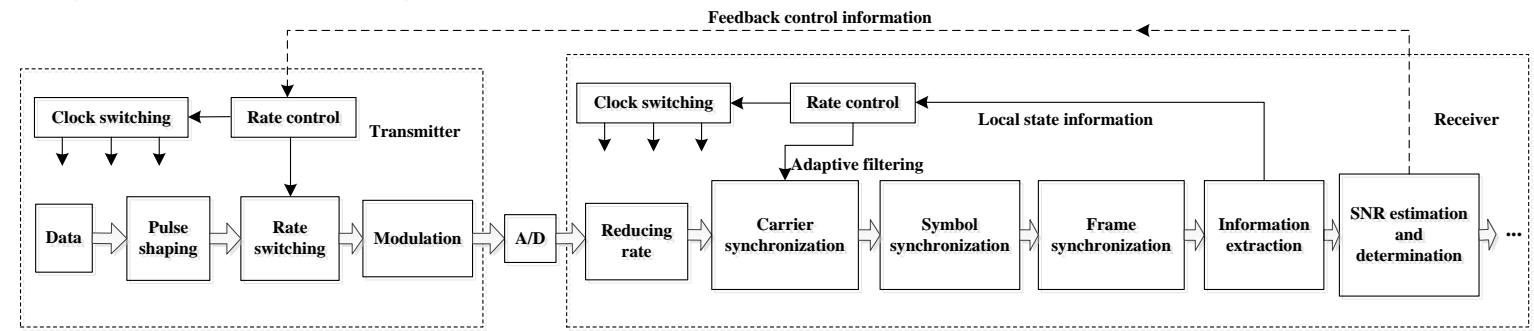

Fig. 1 Adaptive rate transmission scheme via SNR

\section{SNR estimation in adaptive transmission}

This paper intends to employ DF estimation method and adjust bit rate based on SNR in the receiver and communication distance. The received signal of QPSK can be represented as [2]

$$
r_{k}=s_{k}+n_{k}=A a_{k}+n_{k}
$$

where $s_{k}$ is the real constellation signal, $A$ is the amplitude of the signal, $a_{k} \in\{1+\mathrm{j}, 1-\mathrm{j},-1+\mathrm{j},-1-\mathrm{j}\}$, $n_{k}$ is zero-mean complex Gaussian white noise, the variance of the real part and the imaginary part is equal to $\sigma^{2}$. The received signal can be decomposed into two parts

$$
r_{k_{-} I / Q}= \pm A+n_{k}
$$

The SNR of the received signal of QPSK can be obtained by [9]

$$
\begin{aligned}
S N R & =\frac{\left|s_{k}\right|^{2}}{2 \sigma^{2}}=\lambda=\frac{\operatorname{real}\left(s_{k}\right)^{2}+\operatorname{imag}\left(s_{k}\right)^{2}}{2 \sigma^{2}} \\
& =\frac{A^{2}}{\sigma^{2}}=S N R_{I / Q}
\end{aligned}
$$

where real(.) and $\operatorname{imag}($.$) are the real part and the imaginary part, respectively. Thus, the SNR of the$ whole complex signal is equal to that of real part or imaginary part, and only one part of SNR is needed to be estimated. Assume that the signal and noise are mutually independent and we have

$$
\begin{aligned}
E\left(r_{k_{-} I / Q}^{2}\right) & =E^{2}\left(r_{k_{-} I / Q}\right)+\operatorname{var}\left(r_{k_{-} I / Q}\right) \\
= & A^{2}+\sigma^{2}
\end{aligned}
$$

Let

$$
Z=\frac{E\left[r_{k_{-} I / Q}^{2}\right]}{E^{2}\left[\left|r_{k_{-} I / Q}\right|\right]}
$$

where

$$
E\left(\left|r_{k_{-} I / Q}\right|\right)=\sigma \sqrt{\frac{2}{\pi}} \mathrm{e}^{-\left(\frac{A^{2}}{2 \sigma^{2}}\right)}+A\left[\operatorname{erf}\left(\sqrt{\frac{A^{2}}{2 \sigma^{2}}}\right)\right]
$$

Therefore,

$$
z=\frac{1+\lambda}{\left\{\sqrt{\frac{2}{\pi}} \mathrm{e}^{-\left(\frac{\lambda}{2}\right)}+\sqrt{\lambda}\left[\operatorname{erf}\left(\sqrt{\frac{\lambda}{2}}\right)\right]\right\}^{2}}=f(\lambda)
$$

The value can be acquired by estimating the mean of the square of the signal and the absolute value of the signal. Now $\lambda=g(z)$, considering the complexity of the calculation, it is hard to get the closed-form solution. But through data fitting method, it can be approximated by polynomial in the $\lambda$ span of time.

$$
\lambda=\sum_{i=0}^{I} a_{i} z^{i}
$$


where $a_{i}, i=1, \cdots, I$ is the multinomial coefficient.

The estimation of $z$ can be acquired by average estimation of a piece of data, namely

$$
z \approx \frac{E\left(r_{k_{-} I}{ }^{2}\right)}{E^{2}\left(\left|r_{k_{-} I}\right|\right)} \approx \frac{E\left(r_{k_{-} Q}{ }^{2}\right)}{E^{2}\left(\left|r_{k_{-} Q}\right|\right)} .
$$

For QPSK signal, the use of real part or imaginary part can estimate result, but the useful signal is apparently not well utilized. So Eq. (9) can be used to estimate the value of $z$.

$$
\hat{z}=\frac{E\left({r_{k_{-} I}}^{2}\right)+E\left(r_{k_{-} Q}{ }^{2}\right)}{E^{2}\left(\left|r_{k_{-} I}\right|\right)+E^{2}\left(\left|r_{k_{-} Q}\right|\right)}
$$

The complexity of M2M4, SVR, SNV and DF algorithms is compared and shown in Table. 1, where $L$ is the number of samples. It can be seen that DF estimation algorithm has the lowest complexity in Table. 1.

Table. 1 Complexity analysis

\begin{tabular}{|c|c|c|c|c|}
\hline Algorithms & M2M4 method & SVR method & SNV method & DF method \\
\hline $\begin{array}{c}\text { Complexity/The number } \\
\text { of multiplication }\end{array}$ & $5 L$ & $9 L$ & $8 L$ & $4 L$ \\
\hline
\end{tabular}

\section{Simulation Results}

We use Monte Carlo simulations to assess the performance of the M2M4 algorithm, SVR algorithm, SNV algorithm and DF algorithm. In simulations, the noise is Gauss white noise, the channel is AWGN channel, the modulation mode is QPSK. Figs. 2-3 present the estimation of the algorithms for QPSK signal in AWGN channel. It is indicated that the estimation of DF algorithm is better than others when SNR $<0 \mathrm{~dB}$ and almost same to others when $\mathrm{SNR} \geq 0 \mathrm{~dB}$. Furthermore, the computation complexity of DF algorithm is the least among these algorithms.

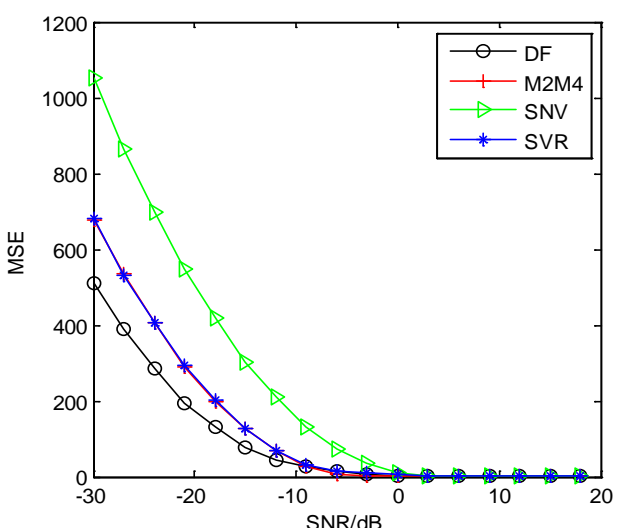

Fig. 2 Estimation comparison $(L=300)$

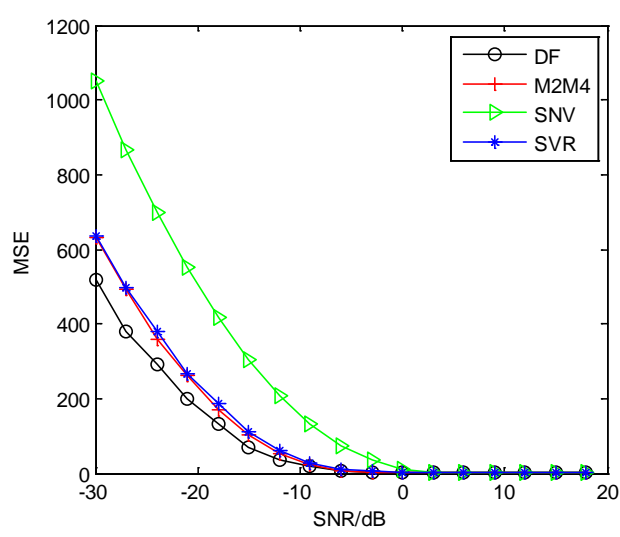

Fig. 3 Estimation comparison $L=500$ )

\section{Conclusions}

In the paper, we use SNR as the standard to measure the channel state, and obtain the SNR estimation to change the transmission rate. We employ DF algorithm for SNR estimation, which is 
key technology of the adaptive transmission. The algorithm has low computational complexity, and has better SNR estimation performance than other algorithms.

Acknowledgement: This work is supported by China NSF Grants (61371169), Qing Lan Project, and priority academic program development of Jiangsu high education institutions.

\section{References}

[1] $\mathrm{Ru} \mathrm{J} \mathrm{X.} \mathrm{Numerical} \mathrm{analysis} \mathrm{of} \mathrm{Mars} \mathrm{exploration} \mathrm{orbit[J].} \mathrm{Science} \mathrm{in} \mathrm{China} \mathrm{(Series} \mathrm{E:}$ Technological Sciences), 2009, 39(03): 528-534.

[2] Fu W J, Jiang J S, Wang X R, Liu S Y. New SNR Estimation Methods in Digital QPSK Receiver[J]. Journal of Electronics \& Information Technology, 2007, Vol29, No.2, 255-258.

[3] Wiesel A, Goldberg J, Messer H. Non-Data-Aided Signal-to-Noise-Ratio Estimation[C]// 2002 IEEE International Conference on Communications, IEEE, 2002:197-201.

[4] Li B, Difazio R, Zeira A. A low bias algorithm to estimate negative SNRs in an AWGN channel[J]. IEEE Communications Letters, 2002, 6(11):469-471.

[5] Shah B, Hinedi S. The split symbol moments SNR estimator in narrow-band channels[J]. IEEE Transactions on Aerospace \& Electronic Systems, 1990, 26(5):737-747.

[6] M. K. Simon and S. Dolinar. Signal-to-noise ratio estimation for autonomous receiver operation[J]. IEEE GLOBECOM’04, pp: 282-287 Dallas, TX, 2004.

[7] Tugnait J K, Gummadavelli U. Blind channel estimation and deconvolution in colored noise using higher-order cumulants[C]// Advanced Signal Processing: Algorithms, Architectures, and Implementations, 1994:106-125.

[8] Matzner R, Englberger F. An SNR estimation algorithm using fourth-order moments[C]// 1994 IEEE International Symposium on Information Theory, IEEE, 1994.

[9] Ren G L, Chang Y L, Zhang H. A new SNR estimator for QPSK Modulation in an AWGN channel [J]. IEEE Transactions Circuits and Systems II: Express Briefs, 2005, 52(6): 336-338.

[10]Salman T, Badawy A, Elfouly T.M, Khattab T. Non-data-aided SNR Estimation for QPSK Modulation in AWGN Channel[C]. IEEE Wireless and Mobile Computing, Networking and Communications Conference, Larnaca, 2014:611-616.

[11]Hua Xu, Zupeng Li, Hui Zheng. A non-data-aided SNR estimation algorithm for QAM signals[C]. IEEE Circuits and Systems, International Conference on Communications, Chengdu, 2004: 999-1003. 